Rev. salud pública. 14 (1): 102-115, 2012

\title{
Curso clínico y pronóstico a un año de una cohorte de prematuros dados de alta con oxígeno domiciliario en Bogotá, Colombia
}

\section{The clinical course and one year forcast for a cohort of premature infants who were discharged with home oxygen in Bogota, Colombia}

\author{
Nathalie Charpak ${ }^{1}$, Juan Gabriel Ruiz ${ }^{2}$ y Sylvia Motta ${ }^{1}$
}

1 Fundación Canguro, Bogotá, Colombia.ncharpak@gmail.com, herchar5@colomsat.net.co, sylvia. helena.motta@gmail.com

2 Departamento de Epidemiología Clínica y Bioestadítica. Pontificia Universidad Javeriana. Bogotá, Colombia.jruiz@javeriana.edu.co

Recibido 17 Mayo 2011/Enviado para Modificación 16 Feberero 2012/Aceptado 25 Febrero 2012

\section{RESUMEN}

Objetivo Documentar el curso clínico y pronóstico de una cohorte de niños prematuros dados de alta con oxígeno domiciliario en Bogotá, Colombia.

Método Cohorte prospectiva de 194 prematuros de 34 o menos semanas de edad gestacional (EG) al nacer, egresados de 12 unidades neonatales con oxígeno suplementario, seguidos hasta la edad corregida de 12 meses para determinar supervivencia, crecimiento, desarrollo y morbilidad.

Resultados 49 niños (25,3\%) tenían una oxigenodependencia leve y $145(74,7 \%)$ moderada-severa. Se presentaron 3 muertes (1,5\%), el estado vital a 40 semanas de EG fue conocido en 169 pacientes (87,1\%) y en 103 (53\%) a 12 meses. La lactancia materna fue exitosa en 147 (75,8 \%) niños a 40 semanas. La antropometría al año fue: peso $8991 \mathrm{~g}$, talla $73 \mathrm{~cm}$ y perimetro craneano $46,2 \mathrm{~cm}$. A las 40 semanas y a 3 meses $74,1 \%$ y $22,7 \%$ de la cohorte aún utilizaba oxígeno el cual fue descontinuado en promedio a los109 días postnatales. El 56,8 \% de los niños tuvo al menos un reingreso y $47 \%$ por patologías respiratorias. Sólo se tamizó para retinopatía al $71 \%$ y en ellos la incidencia de retinopatía de la prematurez (ROP) fue $38 \%$ con 3 cirugías y 1 niño ciego. Un tamizado neuropsicomotor y sensorial se realizó solamente en $19 \%$ encontrando algún tipo de alteración neuropsicomotriz en $30 \%$, refractiva en $40 \%$ y auditiva en $5 \%$.

Conclusión Más del $60 \%$ de los niños oxígeno-dependientes fueron dados de alta sin plan de seguimiento estructurado. El problema de los niños oxigenodependientes es complejo y nuestros datos sugieren un gran espacio para mejorar el seguimiento.

Palabras Clave: Recien nacido, prematuro, atención ambulatoria, displasia broncopulmonar, método madre-canguro, estudios de seguimiento (fuente: DeCS, BIREME). 


\section{ABSTRACT}

Objective Documenting the clinical course and forecast for a concurrent cohort of discharged preterm infants who received home oxygen in Bogota,Colombia.

Methods This was a prospective study of a concurrent cohort of 194 newborn infants having 34 weeks gestational age (GA) or less at birth who were born in 12 institutions and followed up for one year of corrected age to assess mortality, morbidity, growth and development.

Results Oxygen dependency was mild in 49 infants $(25.3 \%)$ and moderate-severe in 145 of them $(74.7 \%)$. There were 3 deaths; vital status was known in 169 infants at 40 weeks GA $(87.1 \%)$ and $103(53 \%)$ at 1 year. Breast feeding at term was successful in $147(75.8 \%)$ infants. Growth indices at one year were appropriate (8,991 g weight, $73 \mathrm{~cm}$ height and $46.2 \mathrm{~cm}$ head circumference) $74.1 \%$ of the cohort were still receiving home oxygen at 40 weeks and and $22.7 \%$ at 3 months and oxygen was discontinued on average on postnatal day $109.56 .8 \%$ of the cohort were readmitted to hospital at least once, $47 \%$ of them because of respiratory conditions. Only $71 \%$ had ophthalmological screening and retinopathy of prematurity (ROP) was detected in $38 \%$ of cases (4 severe cases: 3 laser surgery and 1 blind infant). Neuro-psychomotor and sensorial screening tests were only performed on $19 \%$ of the infanys.

Conclusion More than $60 \%$ of newborn infants discharged with home oxygen lacked structured follow-up. Oxygen-dependancy in infants is complex; our data suggested that there is plenty of room for improvement in Bogotá in that respect.

Key Words: Infant, premature, ambulatory care, bronchopulmonary dysplasia, kangaroo-mother care, follow-up studies (source: MeSH, NLM).

$\mathrm{L}$ a característica fundamental de la enfermedad pulmonar crónica del prematuro, o displasia broncopulmonar (DBP), es el requerimiento de oxígeno por largos períodos de tiempo. Así, los estimativos de la incidencia de la enfermedad están basados en la frecuencia de la dependencia de oxígeno y la clasificación aceptada de su severidad está basada en la severidad de la dependencia de oxígeno (Cuadro 1).

En Bogotá, los niños prematuros son tratados en unidades de recién nacidos (URN) públicas o privadas. En muchas instituciones, tan pronto como el niño es estabilizado y se acerca a su salida, es atendido por medio del Método Madre Canguro (MMC). La posición canguro (contacto piel a piel permanente), junto con una estrategia de nutrición apropiada y un adecuado entrenamiento materno, permite el egreso de los niños prematuros que no regulan temperatura, sean o no oxígeno dependientes, siempre y cuando se garantice un seguimiento cercano y estricto en una consulta ambulatoria estructurada llamada "Programa Madre Canguro" (PMC). 


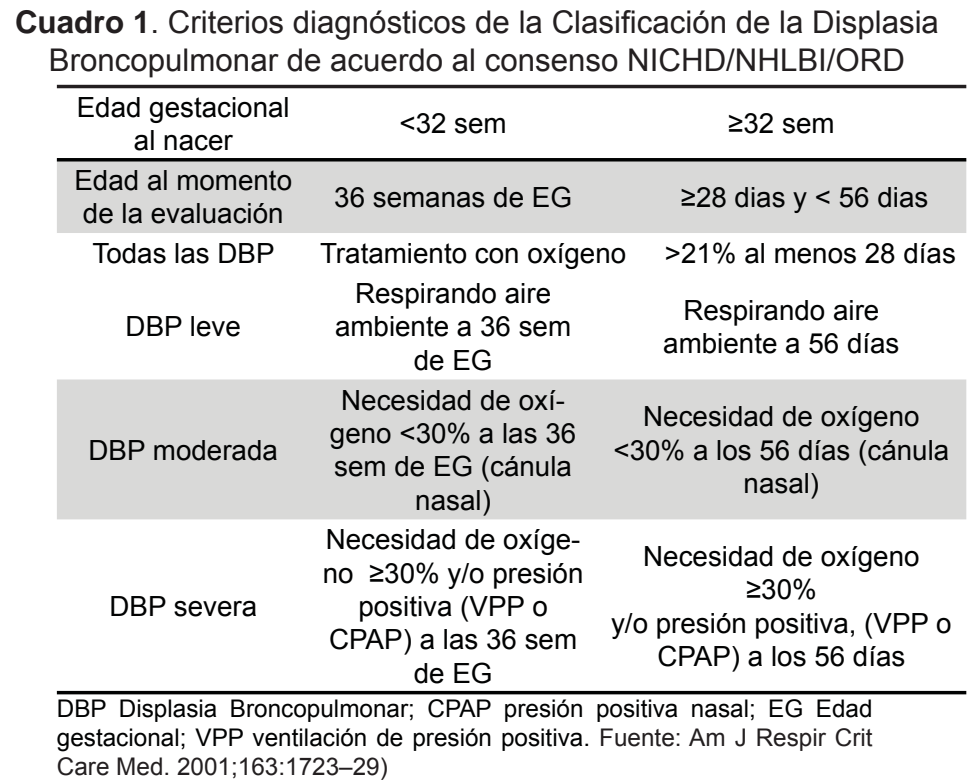

Estadísticas vitales (1994-2005) no publicadas de dos PMC en Bogotá, mostraron que en comparación con los años 90 , el número de ingresos con oxígeno domiciliario en los PMC aumentó de manera importante durante la última década (a partir del 2000). La mayoría de estos niños recibió oxígeno por más de 28 días lo que los clasifica como DBP. Este incremento no parecía ser debido únicamente al aumento en la supervivencia de los niños prematuros con mayor riesgo para la enfermedad (más imaduros) sino se observaba también en recién nacidos más maduros (32 a 34 semanas de edad gestacional al nacer).

Con el propósito de esclarecer estas observaciones, llevamos a cabo un estudio de cohorte prospectiva para estimar de manera confiable y no sesgada las incidencias de oxígeno dependencia y displasia broncopulmonar en niños prematuros en Bogotá (datos publicados en otro artículo). El curso clínico y pronóstico de los niños dados de alta con oxígeno suplementario son presentados en este documento.

\section{MÉTODOS}

Diseño y muestra

Del 01/04/04 al 01/10/04, se reclutó una muestra secuencial de 516 recién 
nacidos prematuros vivos ( $\leq 34$ semanas de edad gestacional al nacer), admitidos en 12 URN de Bogotá. De ellos, 20 niños fueron remitidos y 496 quedaron elegibles para el estudio, 80 murieron antes de cumplir 28 dias de vida, dejando 416 niños candidatos a desarrollar una DBP, 11 murieron antes de la salida, 21 se clasificaron como DBP y se destetaron antes de la salida, 190 salieron sin oxígeno y 194 salieron con oxígeno domiciliario.

En el presente estudio se realizó el seguimiento de esta sub-cohorte prospectiva de 194 prematuros supervivientes con DBP dados de alta con oxígeno domiciliario. Todos fueron seguidos hasta determinar la severidad de la DBP: leve versus moderada-severa (se fusionaron las categorías moderada y severa). Se obtuvo consentimiento informado escrito por parte de los padres, y el Comité de Ética de la Facultad de Medicina de la Pontificia Universidad Javeriana aprobó el protocolo de investigación.

Procedimiento

Las familias de los niños dados de alta con oxígeno fueron invitadas a participar en el estudio. Se tomó la decisión de no centralizar el seguimiento, y observar en el contexto de salud actual en Colombia: 74 niños $(37,4 \%)$ fueron seguidos en Programas Madre Canguro (PMC) con seguimiento ambulatorio estructurado y la información fue obtenida directamente de sus registros. El resto de los participantes asistieron a consultas de seguimiento no estructuradas (CNE) (centros de atención de sus aseguradoras, proveedores privados ocasionales o centros de salud). En estos casos, las madres fueron contactadas telefónicamente y se solicitó la información sobre las evaluaciones clínicas a 40 semanas de edad gestacional y a 3,6, 9 y 12 meses de edad corregida de acuerdo con el seguimiento periódico recomendado por el Ministerio Colombiano de la Protección Social para los niños de bajo peso al nacer (1).

El manejo clínico fue realizado por pediatras en todos los PMC. En las CNE el personal de atención era más diverso, desde pediatras hasta enfermeras profesionales. La heterogeneidad se debe a los múltiples sistemas de aseguramiento de la salud que tienen contratos con las instituciones participantes, así como a la movilidad de los pacientes dentro del sistema de seguridad social de salud. 
Variables

Fueron evaluados los estimativos de mortalidad, duración total de la suplementación con oxígeno, índices de crecimiento y desarrollo, reingresos, estancia hospitalaria y prevalencia de las secuelas como indicadores del curso clínico y pronóstico. Se evaluó también qué tan completa era la información disponible.

Análisis de datos

La información fue recolectada en formatos pre-codificados y la limpieza de los datos se realizó de manera manual y electrónica. Se calcularon estadísticas descriptivas incluyendo medidas de tendencia central y de dispersión para las variables contínuas, proporciones para las variables discretas, e intervalos de confianza del $95 \%$ como medidas de precisión.

\section{RESULTADOS}

Descripción de la cohorte

En 49 de los niños (25,3\%) la oxígenodependencia se debió a DBP leve y en los 145 restantes $(74,7 \%)$ a DBP moderada/severa. Las familias participantes tenían un ingreso percápita mensual de 252.000 pesos, y $18 \%$ de las madres y $12,6 \%$ de los padres eran analfabetas. Solamente el $48 \%$ de las madres asistió a control prenatal durante el primer trimestre y 8,7 \% tuvieron embarazo múltiple. La razón masculino:femenino de los niños fue balanceada. Al nacer, los promedios de peso fueron de $1407 \mathrm{~g}(600-2320)$, talla de $39,6 \mathrm{~cm}(25,5-$ 48) y perímetro craneano (PC) de $31,1 \mathrm{~cm}(23-34)$ y la edad gestacional osciló entre 23 y 34 semanas (promedio 31,1 sem). Los puntajes de Apgar al minuto mostraron algún grado de asfixia neonatal en $65 \%$ de los niños, y el $25 \%$ tuvieron puntajes sub-estándar a los 5 minutos (Tabla 1).

\section{Hospitalización}

El promedio de duración de hospitalización fue 39 días y $93 \%$ de los pacientes pasaron por la unidad de cuidados intensivos en algún momento. El peso al nacer fue recuperado alrededor del día 15 . Los niños con DBP sola, tuvieron una estancia hospitalaria promedio de 40 días $( \pm 21)$, mientras que los niños que presentaron hipertensión pulmonar asociada estuvieron en promedio 50 días ( \pm 29$)$. El diagnóstico radiológico de DBP se realizó únicamente en el 20,9 $\%$ de los niños. Más del $80 \%$ de los pacientes tuvieron una ecografía cerebral, y en $30 \%$ de ellos se encontró algún grado de hemorragia intraventricular (grado I en $16 \%$ de los casos). Los niños recibieron oxígeno por 37 días en 
promedio (7-158) antes del egreso. La duración media de ventilación mecánica fue de 7 días (0-94). La distribución de la severidad de la DBP se observa en la Tabla 2.

Tabla 1. Características al nacer y al egreso de los 194 prematuros de la muestra

\begin{tabular}{|c|c|c|}
\hline & Categoría & $\mathrm{N}(\%)$ \\
\hline \multirow{7}{*}{ Peso al nacer categorizado(g) } & $<701$ & $2(1)$ \\
\hline & $701-1000$ & $26(13,4)$ \\
\hline & $1001-1200$ & $38(19,6)$ \\
\hline & $1201-1200$ & $54(27,8)$ \\
\hline & $1501-1800$ & $46(23,7)$ \\
\hline & $1801-2000$ & $12(6,2)$ \\
\hline & $>2000$ & $16(8,2)$ \\
\hline \multirow{4}{*}{$\begin{array}{l}\text { Edad gestacional categorizado } \\
\text { (sem) }\end{array}$} & $<29$ & $32(16,5)$ \\
\hline & $29-30$ & $36,5(18,6)$ \\
\hline & $31-32$ & $60(30,9)$ \\
\hline & $33-34$ & $66(34)$ \\
\hline $\begin{array}{l}\text { *Lubchenco al nacer } \\
\text { Prematuro con peso adecuado } \\
\text { Prematuro con bajo peso }\end{array}$ & & $\begin{array}{l}148(76,5) \\
46(23,7)\end{array}$ \\
\hline $\begin{array}{l}\text { Patrones de alimentación } \\
\text { LM exclusiva al egreso } \\
\text { LM y Formula } \\
\text { Formula exclusiva }\end{array}$ & & $\begin{array}{l}53,4 \\
40,4 \\
6,2\end{array}$ \\
\hline \multicolumn{3}{|l|}{ Tipo de manómetro al egreso } \\
\hline Adulto & & $14(7,2)$ \\
\hline Neonatal & & $4(2,1)$ \\
\hline Pediatrico & & $96(49,5)$ \\
\hline Datos faltantes & & $80(41,2)$ \\
\hline
\end{tabular}

Características de egreso

Al egreso los promedios de peso fueron de $1950 \mathrm{~g}$ (1320-3700), talla $41,8 \mathrm{~cm}$ (38-45) y PC $31 \mathrm{~cm}$ (27-38) y la edad gestacional osciló entre 32 y 52 semanas (promedio 36,7 sem). Los observadores verificaron la presencia de hipoxemia en el momento del egreso, definida como saturación de la hemoglobina $<90 \%$ al respirar el aire ambiente $(2,3)$ durante una medición dinámica (evaluación en reposo, durante la succión y el sueño). La estancia hospitalaria promedia fue de 39 dias (7-157). La edad gestacional de los niños con DBP leve con y 
sin hipertensión pulmonar (32,8 semanas) fue mayor que la de los niños con DBP moderada o severa (30,5 semanas). El peso promedio de los niños con DBP moderada o severa (con o sin hipertensión pulmonar), fue más bajo que el de aquellos con DPB leve: 1300 g vs. 1600 g. Es importante anotar que solamente un número pequeño de las balas de oxígeno tenían un manómetro neonatal que permite la administración de flujos de oxígeno de menos de $1 / 2$ litro y un destete progresivo del oxígeno (Tabla1).

Tabla 2. Distribución de la cohorte de acuerdo a la severidad de la DBP, la edad gestacional y el peso al nacer

\begin{tabular}{|c|c|c|c|}
\hline & & \multicolumn{2}{|c|}{ Severidad de la DBP } \\
\hline & & DBP leve (\%) & $\begin{array}{c}\text { DBP moderada/severa } \\
(\%)\end{array}$ \\
\hline \multirow{7}{*}{ Peso al nacer } & hasta $700 \mathrm{~g}$ & 0,0 & 100,0 \\
\hline & $707 \mathrm{~g}$ a $1000 \mathrm{~g}$ & 3,8 & 96,2 \\
\hline & $1001 \mathrm{~g}$ a $1200 \mathrm{~g}$ & 18,4 & 81,6 \\
\hline & $1201 \mathrm{~g}$ a $1500 \mathrm{~g}$ & 18,5 & 81,5 \\
\hline & $1501 \mathrm{~g}$ a 1800 & 41,3 & 58,7 \\
\hline & $1801 \mathrm{~g}$ a $2000 \mathrm{~g}$ & 50,0 & 50,0 \\
\hline & 2001g y Más & 37,5 & 62,5 \\
\hline \multirow{4}{*}{$\begin{array}{l}\text { Edad gestacional } \\
\text { al nacer }\end{array}$} & $\begin{array}{l}\text { hasta } 28 \text { sema- } \\
\text { nas }\end{array}$ & 9,4 & 90,6 \\
\hline & 29 a 30 semanas & 2,8 & 97,2 \\
\hline & 31 a 32 semanas & 28,3 & 71,7 \\
\hline & 33 a 34 semanas & 42,4 & 57,6 \\
\hline \multirow{2}{*}{ Total } & $\mathrm{N}$ & 49 niños & 145 niños \\
\hline & $\%$ & 25,3 & 74,7 \\
\hline
\end{tabular}

Crecimiento y desarrollo durante el seguimiento

El crecimiento somático en general fue adecuado. A 40 semanas y a 12 meses de edad corregida los valores promedios fueron respectivamente: $2918 \mathrm{~g}$ y $8991 \mathrm{~g}$ para el peso, $47,2 \mathrm{~cm}$ y $73,2 \mathrm{~cm}$ para la talla y $35 \mathrm{~cm}$ y $46,2 \mathrm{~cm}$ para el PC. No se encontraron diferencias en los índices de crecimiento somático en los niños con diferentes tipos de oxígeno dependencia, excepto por el promedio de peso a 12 meses de edad corregida, que fue menor para los niños con hipertensión pulmonar asociada.

La información sobre el desarrollo neuro y psicomotor solamente estuvo disponible para los niños que fueron atendidos en los PMC, en donde se realizó una evaluación sistemática del tono muscular a las 40 semanas de edad gestacional en 39 niños: 29 normales, 9 hipotónicos y 2 hipertónicos. A 
los 3, 6, 9 y 12 meses de edad corregida, se realizó un tamizado neurológico para identificar a los niños que podían necesitar una evaluación más detallada y ser remitidos a terapia física específica. La prueba utilizada clasifica a los niños en tres grupos: evaluación neuromotora "normal, "transitoria" y "anormal". A los 3 meses, se contaba con información para 60/74 (81 \%) niños, $13(22 \%)$ fueron clasificados como transitorios y niguno tuvo un resultado anormal. El coeficiente de desarrollo psicomotor promedio fue de $98 \pm 7,5$ (DE) a los 6 meses y a los 12 meses fue de 101 $\pm 5,3$ en 52 niños. De estos niños, 4 presentaron un retraso psicomotor leve.

Alimentación

Llegando a las 40 semanas, $26,2 \%$ de los niños recibía lactancia materna exclusiva y a los 3 meses $44,2 \%$ tenía lactancia materna total o parcial Esta proporción disminuyó de manera dramática a la edad de 6 meses: solamente el $18,9 \%$ de las madres contínuaba ofreciendo algo de leche materna a los niños.

Evolución de la oxígeno dependencia

El oxígeno fue suspendido a 106 días o antes ( 1 mes de edad corregida) en 168 niños, y solamente el $16 \%$ continuó recibiendo oxígeno después de los 3 meses de edad corregida. El oxígeno suplementario fue administrado por un promedio de 101 días (14-506), y solo 1 niño continuaba recibiendo oxígeno al final del seguimiento a los 12 meses. La edad gestacional al destete del oxígeno fue variable de acuerdo a la severidad de la DBP: 38,5 semanas(33,3-41,4) en las DBP leves y 48,4 semanas $(33,3-103,4)$ en las DBP moderada-severas.

Otra variable que afectó de manera significativa la duración de la administración de oxígeno fue el tipo de programa en el que los pacientes eran seguidos (PMC vs. CNE). Se encontraron diferencias significativas entre el PMC y los CNE en la duración del oxígeno suplementario y la edad gestacional al destete del oxígeno: 86 días y 118 días respectivamente ( $\mathrm{p}=0,027$, ANOVA-ANCOVA), incluso después de controlar los datos por edad gestacional al nacer (Tabla 3 ) 
Tabla 3. Edad gestacional al destete de oxígeno de acuerdo a la edad gestacional al nacer y tipo de seguimiento

\begin{tabular}{|c|c|c|c|c|}
\hline \multirow{2}{*}{$\begin{array}{c}\text { Edad gestacional } \\
\text { al nacer }\end{array}$} & \multirow{2}{*}{$\begin{array}{l}\text { Programa de } \\
\text { seguimiento }\end{array}$} & \multirow{2}{*}{ Media } & \multicolumn{2}{|c|}{ IC 95\% } \\
\hline & & & Limite Inf & Limite sup \\
\hline \multirow[t]{2}{*}{ Hasta 28 semanas } & No estructurado & 52,257 & 46,700 & 57,814 \\
\hline & $\begin{array}{l}\text { Estructurado } \\
\text { (PMC) }\end{array}$ & 49,551 & 43,581 & 55,520 \\
\hline \multirow[t]{2}{*}{29 a 30 semanas } & No estructurado & 50,048 & 44,491 & 55,605 \\
\hline & $\begin{array}{l}\text { Estructurado } \\
\text { (PMC) }\end{array}$ & 48,042 & 42,822 & 53,262 \\
\hline \multirow[t]{2}{*}{31 a 32 semanas } & No estructurado & 46,577 & 42,509 & 50,644 \\
\hline & $\begin{array}{l}\text { Estructurado } \\
\text { (PMC) }\end{array}$ & 42,714 & 38,125 & 47,302 \\
\hline \multirow[t]{2}{*}{33 a 34 semanas } & No estructurado & 46,543 & 42,238 & 50,847 \\
\hline & $\begin{array}{l}\text { Estructurado } \\
\text { (PMC) }\end{array}$ & 43,103 & 39,298 & 46,907 \\
\hline
\end{tabular}

Mortalidad y morbilidad

Tres niños murieron durante el seguimiento, todos durante reingresos hospitalarios prolongados que ocurrieron antes de los 6 meses de vida.

Durante el primer control ambulatorio (a las 40 semanas de edad gestacional), no se contaba con información para el $13 \%$ de los niños. A los 12 meses, la información se obtuvo únicamente para el 53 \%. La alta movilidad de esta población (cambios de domicilio y de proveedores de salud) fue la principal limitación para el seguimiento. Hubo menos deserciones y perdidos de vista en los programas estructurados (PMC).

El $73 \%$ de los niños se rehospitalizó al menos 1 vez y en el $67 \%$ de los casos, por enfermedad pulmonar. Estos pacientes acumularon en promedio 12 días de estancia por reingreso y 20 visitas ambulatorias hasta el año de edad corregida. El número total y la duración de los reingresos fue diferente para los niños atendidos en los PMC (con protocolos de manejo explícitos) en comparación con los CNE. Después de controlar con ANOVA-ANCOVA por la severidad de la DBP, el número promedio de reingresos fue 0,6 por niño por año en los PMC y 1,9 por niño por año para los $\mathrm{CNE}(\mathrm{p}=0,02)$. El promedio de estancia acumulada fue de 4,6 días en los PMC y 18,1 días en los CNE $(\mathrm{p}=0,016)($ Tabla 4$)$.

El $12 \%$ se rehospitalizó por anemia y transfusión antes de alcanzar las 40 semanas de edad gestacional. 
Tabla 4. Estancia hospitalaria acumulada (días) durante el primer año según la edad corregida y el tipo de seguimiento

\begin{tabular}{ccccccc}
\hline \multicolumn{1}{c}{ CNE } & Media & $\begin{array}{c}\text { Hasta 40 } \\
\text { semanas }\end{array}$ & $\begin{array}{c}\text { Hasta 3 } \\
\text { meses }\end{array}$ & $\begin{array}{c}\text { Hasta 6 } \\
\text { meses }\end{array}$ & $\begin{array}{c}\text { Hasta 9 } \\
\text { meses }\end{array}$ & $\begin{array}{c}\text { Hasta 12 } \\
\text { meses }\end{array}$ \\
\hline \multirow{2}{*}{ PMC } & DE & 10,45 & 8,89 & 10,38 & 13,70 & 18,41 \\
\hline & Media & 1,00 & 21,18 & 17,33 & 20,02 & 26,46 \\
\hline & DE & 3,45 & 5,92 & 5,43 & 5,66 & 10,45 \\
\hline
\end{tabular}

CNE: Programas no estructurados; PMC: programas estructurados

A pesar de que la retinopatía de la prematurez (ROP) se asocia al uso de oxígeno, solo en 137 niños (71 \%), se practicó una evaluación por oftalmólogo. No se encontraron signos de ROP en 85 niños, en 41 niños se encontró ROP de cualquier grado y 3 niños fueron sometidos a cirugía; un caso fue diagnosticado de manera tardía y el paciente quedó ciego.

\section{DISCUSIÓN}

Desde 1989, el MMC ha sido objeto de evaluaciones rigurosas (4-7). En 1993, el primer PMC con seguimiento sistematizado hasta el año de edad corregida fue implementado. El egreso hospitalario en la posición canguro se hizo popular en las aseguradoras de salud públicas y privadas ya que disminuía la estancia hospitalaria (en principio es adecuado y deseable), con la disminución asociada en el riesgo de infección nosocomial y su costoso tratamiento. Después del año 1996, la política de egreso del MMC fue modificada para acomodarla a niños con DBP. Estos niños podían ser dados de alta en posición canguro con bajos flujos de oxígeno ambulatorio para mantener tanto la temperatura como la saturación de oxígeno dentro de parámetros normales. Actualmente hasta el $40 \%$ de los niños admitidos a los PMC ambulatorios en Bogotá son oxígeno dependientes. Esta política de egreso es muy popular debido a que el costo de la provisión de un mes de oxígeno ambulatorio (bala en casa y bala portátil) tiene el mismo costo directo que 1 o 2 días de hospitalización en cuidados mínimos en una URN.

El seguimiento ambulatorio de los niños en posición canguro con oxígeno suplementario es una característica particular del MMC Colombiano y no ha sido reportado en otros países. Sin embargo el seguimiento ambulatorio de niños oxígeno dependientes de alto riesgo durante el primer año es muy similar y ha sido reportado. Así, el manejo domiciliario del oxígeno ha mostrado beneficios en el crecimiento de niños con DBP y en la disminución de muerte súbita. Es por esto que la Academia Americana de Pediatría y la Sociedad Americana del Tórax apoyan esta práctica (8-11). 
La importancia de un equipo multidisciplinario para el manejo de estos infantes frágiles ha sido mencionada (9-11), y el manejo dentro de los PMC es realizado por un equipo con profesionales de diversas especialidades (pediatras, enfermeras, psicólogos, oftalmólogos, terapistas de lenguaje, optómetra, trabajadores sociales). El énfasis en los controles frecuentes iniciales realizados en los PMC hasta lograr al mismo tiempo una estabilización en el crecimiento somático ( $15 \mathrm{~g} / \mathrm{Kg} /$ día $)$ y una adecuada saturación de oxígeno (90-94 \%) y frecuencia cardíaca (en reposo, durante la succión o el sueño) podría explicar los mejores resultados obtenidos en los PMC versus los CNE en este estudio.

El crecimiento somático en esta cohorte de niños oxígeno-dependientes fue apropiado, pero hay que tener en cuenta que el seguimiento hasta el año fue incompleto. La nutrición y el crecimiento somático son preocupaciones centrales para los expertos durante el seguimiento de niños oxígenodependientes (12-14). Aunque la fórmula enriquecida ha mostrado beneficios en el crecimiento y formación ósea en los niños con DBP, en la mayoría de los programas de seguimiento se ha escogido la lactancia materna (con o sin fórmula enriquecida) como la primera opción nutricional por sus beneficios fisiológicos y psicológicos (14). Es así como cerca del $44,2 \%$ de las madres en nuestra cohorte lograron lactar a sus hijos hasta los 3 meses de edad corregida. A los 6 meses la lactancia materna es muy baja y esto se debe en parte a que la duración de la licencia de maternidad en Colombia era de 84 días, sin prolongación para las madres de niños prematuros. Actualmente es de 14 semanas y se prolonga el tiempo que le faltó al niño prematuro para llegar a la semana 37.

De acuerdo con Korhonen y colaboradores $(13,15)$ la tasa de morbilidad en pacientes con DBP es cerca de dos veces mayor que la de los niños prematuros sin DBP. Los niños con DBP ocasionalmente desarrollan hipertensión pulmonar que, como se observó en el presente estudio, exacerba su condición y cualquier episodio de hipoxemia tiene una repercusión inmediata en el crecimiento.

Solamente los niños que fueron seguidos en los PMC tuvieron un reporte de evaluaciones neurológicas, psicomotrices y de desarrollo sensorial. Este hecho es alarmante, ya que los niños con DBP son una población de alto riesgo para retrasos en el desarrollo físico y neurológico (16) y alteraciones en la agudeza visual y auditiva. 
El efecto del oxígeno en la retina prematura es ampliamente conocido, y continúa siendo la principal causa de ceguera en America Latina (17). El cubrimiento de tamizaje para Retinopatía del prematuro en esta cohorte fue de $71 \%$. Esta cifra no es satisfactoria en una población de niños prematuros con oxígeno dependencia a la salida del hospital, como lo era nuestra cohorte.

Estos niños prematuros fueron dados de alta de una URN con una medicación potencialmente peligrosa como lo es el oxígeno contínuo, sin tener programada una supervisión oximétrica completa, oportuna y adecuada. Esta situación surge debido a las grandes diferencias en la estructura y calidad de los programas de seguimiento disponibles para esta población y la falta de protocolos.

El curso clínico y pronóstico de los niños con displasia broncopulmonar y/o hipertensión pulmonar es diferente de acuerdo al programa de seguimiento, que parece ser un factor determinante en el manejo adecuado de estos niños y por lo tanto influencia su pronóstico. En este caso se observó una morbilidad respiratoria mayor y duración de la utilización de oxígeno suplementario más prolongada en los CNE. Hay que enfrentar la problemática de la deserción (en este estudio fue de de casi la mitad) y de deficiente comunicación entre las URN (que dan de alta a estos niños de alto riesgo) y los programas de seguimiento. La dependencia de oxígeno y su manejo racional, particularmente en ambiente ambulatorio, son elementos complejos. El presente estudio sugiere que la estandarización y la racionalización del manejo de estos niños traería beneficios. En nuestro país, los esfuerzos no deben limitarse a la supervivencia de los infantes; deben extenderse a su calidad de vida, al menos durante sus primeros años y no solamente mientras están hospitalizados. Los PMC son actualmente una oportunidad para brindarle a estos niños el seguimiento de alto riesgo que requieren.

Afortunadamente se están elaborando (Ministerio de Salud y Colciencias) protocolos y estándares de calidad para el manejo de los recién nacidos incluyendo lineamientos técnicos canguro (18) que deberían unificar el manejo del recién nacido prematuro y/o de bajo peso al nacer en Colombia

Agradecimientos: Queremos reconocer la cooperación de las unidades de cuidado neonatal de los siguientes hospitales y clínicas: Kennedy, Tunal, Universitario San 
Ignacio, San José, Samaritana, Instituto Materno Infantil, Occidente, Magdalena, Olaya, Partenón, San Pedro Claver y Emmanuel. Un especial reconocimiento a la Dra. Zita Figueroa (†2009) y a la Dra. Lida Rosero de los PMC quienes nos facilitaron los registros de seguimiento de los niños incluidos en este estudio. Este proyecto de investigación fue financiado por el Departamento Administrativo de Ciencia, Tecnología e Innovación, Colciencias. República de Colombia.

Conflicto de intereses: Ninguno

\section{REFERENCIAS}

1. Resolución Ministerio No. 00412 de 2000, Guía de Atención del Bajo Peso al Nacer (5.3) Método Madre Canguro. Diario Oficial Edicion 43956 Marzo 31 de 2000.

2. Lozano JM, Duque OR, Buitrago T, Behaine S. Pulse oximetry reference values at high altitude. Arch Dis Child. 1992;67:299-301.

3. Gamponia MJ, Babaali H, Gilman RH. Reference values for pulse oxymetry at high altitude. Arch Dis Child. 1998;78:461-5.

4. Charpak N, Ruiz JG, Charpak Y. Kangaroo Mother Program. An alternative way for caring for LBW infants? A two cohort study. Pediatrics. 1994;94:804-10.

5. Charpak N, Ruiz-Pelaez JG, Calume Z. Current knowledge in Kangaroo Care. Curr Opin Pediatr. 1996;8:108-12.

6. Charpak N, Calume Z, Ruiz-Pelaez JG, Charpak Y. Kangaroo Mother versus "traditional" care for newborn infants with birth weight $£ 2000 \mathrm{~g}$. A randomized controlled trial. Pediatrics. 1997;100:682-8.

7. Conde-Agudelo A, Belizán JM, Diaz-Rossello J. Kangaroo mother care to reduce morbidity and mortality in low birthweight infants. Cochrane Database Syst Rev. 2011 Mar 16;(3):CD002771.

8. Ellsbury DL, Acarregui MJ, McGuinness GA, Eastman DL, Klein JM. Controversy surrounding the use of home oxygen for premature infants with bronchopulmonary dysplasia. $J$ Perinatol. 2004;24:36-40.

9. American Thoracic Society. Statement on the care of the child with chronic lung disease of infancy and childhood. Am J Respir Crit Care Med. 2003;168:356-96.

10. Bancalari E, Wilson-Costellob D, Ibenc SC. Management of infants with bronchopulmonary dysplasia in North America. Early Hum Dev. 2005;81:171-9.

11. Thomas W, Speer CP. Management of infants with bronchopulmonary dysplasia in Germany. Early Hum Dev. 2005;81:155-63.

12. Atkinson SA. Special nutritional needs of infants for prevention of and recovery from bronchopulmonary dysplasia. J Nutr. 2001;131:942S-6S.

13. Korhonen P, Koivisto AM, Ikonen S, Laippala P, Tammela O. Very low birthweight, bronchopulmonary dysplasia and health in early childhood. Acta Paediatr. 1999;88:1385- 91.

14. Lawrence RA, Lawrence RM. Breastfeeding the infant with a problem. In: Lawrence RA, Lawrence RM (Eds). Breastfeeding, a guide for the medical profession. 6th Ed. St Louis, Missouri: Mosby; 2011. p. 474-514.

15. Smith VC, Zupancic JAF, McCormick MC, Croen LA, Greene J ,Escobar GJ, et al. Rehospitalization in the first year of life among infants with bronchopulmonary dysplasia. J Pediatr. 2004;144:799-803. 
16. Short EJ, Klein NK, Lewis BA, Fulton S, Eisengart S, Kercsmar C, et al. Cognitive and academic consequences of bronchopulmonary dysplasia and very low birth weight: 8 year-old outcomes. Pediatrics. 2003;112:E359-66.

17. Adams RJ, Hall HL, Courage ML. Long-term visual pathology in children with significant perinatal complications. Dev Med Child Neurol. 2005;47:598-602.

18. Lineamientos Técnicos para la Implementación de Programas Madre Canguro en Colombia [Internet]. Disponible: http://www.colombianutrinet.org/index.php?option=com_conten t\&view=article\&id=645:lineamientos-tecnicos-para-la-implementacion-de-programasmadre-canguro-en-colombia-\&catid=118\&Itemid=259 Consultado: Octubre 2011. 